EVOLUTION \& DEVELOPMENT $5: 2,119-120$ (2003)

\title{
The difficulty of agreeing about constraints
}

\author{
Patrícia Beldade* and Paul M. Brakefield \\ Institute of Evolutionary and Ecological Sciences, University of Leiden, P.O. Box 9516, 2300 RA Leiden, The Netherlands \\ *Author for correspondence (e-mail pbeldade@uci.edu)
}

The literature on evolutionary constraints has been characterized by controversies and misunderstandings, many of which result from the difficulty in providing convincing tests of proposed constraints. Our recent experiment (Beldade et al. 2002b) contributed to filling this gap by taking advantage of laboratory population-level studies possible with Bicyclus anynana butterflies (Beldade and Brakefield 2002).

We showed that, despite the genetic correlations and developmental coupling between different eyespots in $B$. anynana, there is much flexibility for independent evolution of eyespot size. Although Arthur (2002, 2003, this issue) was not surprised by our result, we were because it went against our a priori prediction based on clear genetic and developmental data. Response to artificial selection produced all patterns found within the genus and even a candidate "forbidden morphology" (Beldade et al. 2002b). These results suggest, we maintain, that natural selection, rather than any internal constraint, is likely to have a dominant role in shaping diversity in butterfly wing patterns. Arthur (2003, this issue) generalized our result more than we would have dared; we were careful to couple our conclusion to butterfly wing patterns and cautious not to dismiss any potential effect of factors other than natural selection in wing pattern evolution.

We do not believe nor have ever argued that genetic correlations between traits are not an important influence on the direction of multitrait evolutionary change. However, we have shown that for $B$. anynana eyespots, such correlations are unlikely to limit morphological evolution of the patterns of eyespot size. We also do not dispute that the patterns of morphological diversity in nature result from an "interaction" between many types of contingencies (including developmental constraints) and selection (Maynard Smith et al. 1985; Barton and Partridge 2000; Teotónio and Rose 2001). Extreme positions on the debate of constraints versus selection are currently defended by few, and certainly not by ourselves. More experiments are necessary before we can generalize about any relative importance of natural selection and developmental constraints in the wild. In our system, however, given the flexibility our results unraveled, we expect that a history of prolonged natural selection on eyespot size in any particular direction would result in adaptive response, even though change in some directions may be achieved faster than in others (Beldade et al. 2002c).

An important issue is then whether butterfly wing patterns represent a special case or whether the same type of flexibility can be expected for other types of morphologies. Our material, although showing vestiges of the developmental origin of the eyespots as homologous repeated structures (the "ground-plan" idea; Nijhout 2001), is also likely to have experienced a long legacy of natural selection favoring (some) independence of the individual repeats (Beldade et al. 2002c). Even though the same genes are involved in the formation of all eyespots, eyespot-specific gene regulation can facilitate independent evolution of different elements (Beldade et al. 2002a,c).

Butterfly wing patterns lend themselves to an integrated and detailed analysis of constraints. Segment number in centipedes, on the other hand, may well be constrained (Arthur 2003 , this issue), but unfortunately the system is as yet intractable to direct experimental dissection. Furthermore, what is an interesting potential constraint in centipedes is not relevant to butterfly eyespot patterns where, for example, a change in eyespot number can be a by-product of addition or deletion of a wing vein (Brakefield et al. 1996) or of selection for eyespot size (Monteiro et al. 1994; Beldade and Brakefield, 2003, this issue). We sought rather to examine the extent to which individual eyespots could diverge in morphology. In our view there is no real dichotomy between absolute and relative constraints but rather, as so often in biology, a continuum of constraints (limitations/biases) of different strengths. A temporal framework is certainly fundamental in discussions about the relevance of constraints. The wider the coverage of different lineages, the more likely one is to find a candidate "constrained phenotype" not represented in a narrower range of taxa. For example, looking beyond the geophilomorph centipedes to other arthropods, even numbers of leg-bearing segments do occur.

The spectacular diversity in the color patterns decorating butterfly wings is often used to illustrate how "unconstrained" morphological evolution can be. Our data demonstrated the underlying flexibility despite previous suggestions of constraints stemming from genetic and developmental data (Beldade et al. 2002b,c). This approach provides an endorse- 
ment of Arthur's point about the relevance of evo-devo to the study of intrinsic constraints (Arthur 2003, this issue). We believe that more such data are necessary to improve definitions and concepts regarding internal constraints. Claims for constraints, just as those for adaptation (Gould and Lewontin 1979), must be rigorously tested or they risk remaining biological "just-so" stories.

\section{REFERENCES}

Arthur, W. 2002. The interaction between developmental bias and natural selection: from centipede segments to a general hypothesis. Heredity 89: 239-246.

Arthur, W. 2003. Developmental constraint and natural selection. Evol. Dev. 5:117-119.

Barton, N., and Partridge, L. 2000. Limits to natural selection. BioEssays 22: 1075-1084.

Beldade, P., and Brakefield, P. M. 2002. The genetics and evo-devo of butterfly wing patterns. Nat. Rev. Genet. 3: 442-452.

Beldade, P., and Brakefield, P. M. 2003. Concerted evolution and developmental integration in modular butterfly wing patterns. Evol. Dev. 5: 169-179.
Beldade, P., Brakefield, P. M., and Long, A. D. 2002a. Contribution of Distal-less to quantitative variation in butterfly eyespots. Nature 415 : 315-318.

Beldade, P., Koops, K., and Brakefield, P. M. 2002b. Developmental constraints versus flexibility in morphological evolution. Nature 416: 844847.

Beldade, P., Koops, K., and Brakefield, P. M. 2002c. Modularity, individuality, and evo-devo in butterfly wings. Proc. Natl. Acad. Sci. USA 99: 14262-14267.

Brakefield, P. M., Gates, J., Keys, D., Kesbeke, F., Wijngaarden, P. J., Monteiro, A., et al. 1996. Development, plasticity and evolution of butterfly eyespot patterns. Nature 384: 236-242.

Gould, S. J., and Lewontin, R. C. 1979. The Spandrels of San Marco and the Panglossian paradigm: a critique of the adaptationist programme. Proc. R. Soc. Lond. B 205: 581-598.

Maynard Smith, J., Burian, R., Kaufman, S., Alberch, P., Campbell, J., Goodwin, B., et al. 1985. Developmental constraints and evolution. $Q$. Rev. Biol. 60: 265-287.

Monteiro, A. F., Brakefield, P. M., and French, V. 1994. The evolutionary genetics and developmental basis of wing pattern variation in the butterfly Bicyclus anynana. Evolution 48: 1147-1157.

Nijhout, H. F. 2001. Elements of butterfly wing patterns. J. Exp. Zool. 291: 213-295.

Teotónio, H., and Rose, M. R. 2001. Perspective: reverse evolution. Evolution 55: 653-660. 